\title{
Analysis of Coherence-Collapse Regime of Semiconductor Lasers Under External Optical Feedback by Perturbation Method
}

\author{
Qin Zou and Shéhérazade Azouigui \\ Institut Telecom, Telecom SudParis, UMR 5157 CNRS,
}

France

\section{Introduction}

High-performance low-cost semiconductor lasers play a crucial role in development of the future optical-fiber-based telecommunication systems. It is known that the behavior of a semiconductor laser can significantly be altered by external optical feedback, and that this effect can be used for improvement of the performance of a laser such as linewidth narrowing, threshold lowering and intensity-noise reducing. External optical feedback, on the other hand, is also responsible for undesirable phenomena such as mode instability, mode hopping and linewidth broadening, which can severely affect the bit-error rate in data transmission. For this reason, the development of lasers highly resistant to external optical feedback has been the subject of intensive investigations since the last decade.

The tolerance to external optical feedback has been investigated for quantum-well (QW) lasers emitting in the $1.3 \mu \mathrm{m}$ as well as in the $1.55 \mu \mathrm{m}$ wavelength ranges. For applications at $1.3 \mu \mathrm{m}, 350-\mu \mathrm{m}$-long strained multi-quantum-well (MQW)-based antireflection (AR)/high reflectivity (HR) distributed feedback (DFB) lasers with a slope efficiency of $0.3 \mathrm{~W} / \mathrm{A}$ and a threshold of instability of $-15 \mathrm{~dB}$ have been shown to be suitable for $2.5 \mathrm{~Gb} / \mathrm{s}$ transmission without optical isolators under the G.957 International Telecommunication Union recommendation, which specifies a threshold of $-24 d B$ (Grillot et al., 2003). For the $1.55 \mu \mathrm{m}$ band, more sophisticated MQW-based AR/AR chirped-grating DFB lasers were fabricated, with cavities (about $500 \mu \mathrm{m}$ in length) composed of a straight section followed by a stripe section of varying width. These lasers exhibited a threshold of $-22 d B$ obtained with a value of the coupling coefficient equal to $80 \mathrm{~cm}^{-1}$ (Grillot et al., 2004). Considered now as a promising alternative to QW lasers for next-generation optical-fiber networks, quantum-dot (QD) lasers exhibit many interesting properties such as reduced threshold current, low chirp, and weak temperature dependence (Bimberg et al., 1999). Moreover, small values of the linewidth enhancement factor (LEF or $\alpha$ factor) of QD lasers are expected to reduce their sensitivity to external optical feedback. For emission at $1.3 \mu \mathrm{m}$, InAs/GaAs QD lasers have shown a high 
resistance to external optical feedback and a threshold of $-8 d B$ has been obtained for long-cavity $(1.5 \mathrm{~mm})$ devices, resulting from a low LEF value $(\alpha=1.6)$ (O'Brien et al., 2003). For emission in the $1.55 \mu \mathrm{m}$ band, a progress in the growth of InAs/InP quantum dashes (elongated dots, QDashes) (Lelarge et al., 2007) made possible a systematic investigation of the tolerance to feedback for Fabry-Perot (F-P) lasers with controlled values of the LEF and of the differential gain (Azouigui et al., 2007a, 2007b, 2008, 2009). Recently, it was shown that p-type doped InAs/InP QDash DFB lasers are in general more resistant than their F-P counterparts (Zou et al., 2010).

In parallel with experimental investigations, theoretical approaches have also been developed (see for example, Tromborg et al., 1984, Schunk \& Petermann, 1988 and Binder \& Cormack, 1989) in order to understand the nonlinear dynamics of a composite laser system. Good agreement between experiment and theory has been found when this latter is applied in particular to bulk and QW lasers. Most of these methods have been developed on the basis of the model proposed by Lang and Kobayashi (L-K model) (Lang \& Kobayashi, 1980). More recently, asymptotic methods have been applied to modeling of the dynamic properties of a laser subject to optical feedback (Erneux, 2000, 2008). In this approach (called the asymptotic approach for simplicity), a laser under moderate optical feedback is viewed as a weakly-perturbed nonlinear dynamic system and the threshold of instability corresponds to the first Hopf bifurcation of the L-K rate equations.

This chapter investigates a preliminary interpretation of the experimental results recently obtained with InAs/InP QDash F-P lasers (Azouigui et al., 2007b, 2008). It has been found that the behaviors manifested by these lasers, when assessed for their tolerance to optical feedback, can be quite well understood using the formalism developed from the asymptotic approach. In Section 2, we will present briefly this formalism. Section 3 will be devoted to a simple stability analysis of a composite laser system. In Section 4, a qualitative interpretation of experimental results will be made. We will focus on two particular situations: the onset of coherence collapse and the fully-developed coherence collapse (Zamora-Munt et al., 2010). In Section 5, we will make a direct comparison between an analytical expression derived in Section 4 and a widely employed model for determination of the critical feedback level. A conclusion will be given in Section 6 .

\section{Solution of the L-K equations}

In this section, the L-K rate equations will be analytically solved under the assumption of low feedback level. By means of the obtained solutions, the stability of a possible mode of a composite laser system will be completely characterized. We will review the formalism developed in (Erneux, 2000, 2008), outline the main stages of its derivation and introduce some notations that we will use in the following sections.

\subsection{Dimensionless L-K equations}

Consider the configuration of Fig. 1. A single-longitudinal-mode laser diode is in resonance with a F-P resonator. We assume that the mirror $M$ is dispersionless and passive. We also consider small feedback effect (i.e. $R_{2}<<1$ ), so multiple reflections are neglected. 


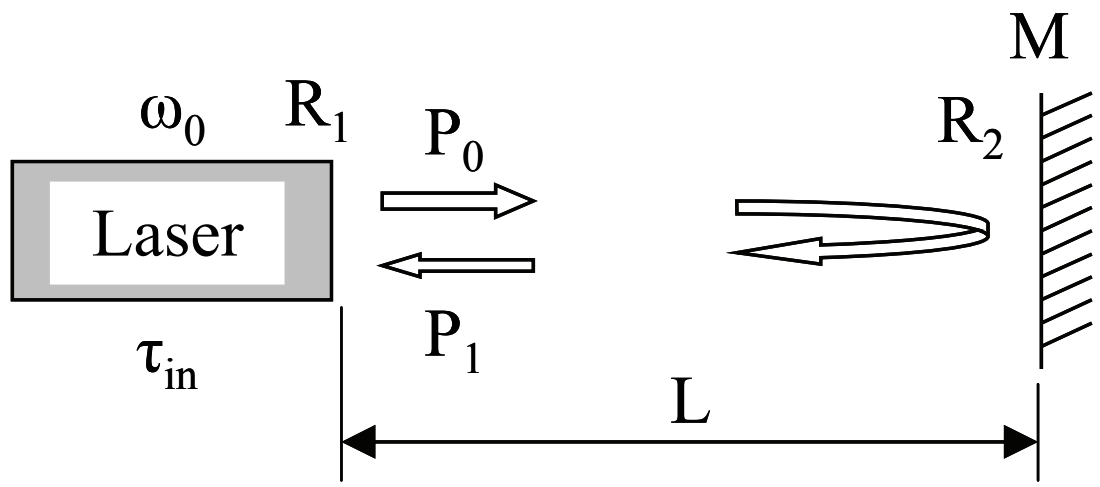

Fig. 1. Schematic drawing of a laser diode with external optical feedback. The external cavity is formed with the output facet of the laser with a reflectivity $R_{1}$ and a distant mirror $M$ with a reflectivity $R_{2}$. $\omega_{0}$ : emission (angular) frequency; $\tau_{\text {in }}$ : internal round-trip time; $P_{0}$ : emission power; $P_{1}$ : feedback power.

The emission behavior of the above composite system is determined by the interference of the initial and time-delayed waves. Under the assumption of weak feedback level, the electric field $E(t)$ and the carrier density $n(t)$ satisfy the L-K rate equations

$$
\begin{gathered}
\dot{E}(t)=\frac{\xi}{2}(1+j \alpha) n(t) E(t)+\gamma E(t-\tau) \exp \left(-j \omega_{0} \tau\right) \\
\dot{n}(t)=J-J_{t h}-\frac{n(t)}{T_{1}}-\left[\Gamma_{0}+\xi n(t)\right]|E(t)|^{2}
\end{gathered}
$$

where $\xi$ (in $s^{-1}$ ) is the differential gain, $\alpha$ is the linewidth enhancement factor (LEF), $\gamma$ (in $s^{-1}$ ) is the feedback rate, $\tau$ (in seconds) is the external delay time, $J-J_{\text {th }}$ (in $s^{-1}$ ) is the pumping current above threshold, $T_{1}$ (in seconds) is the carrier life time and $\Gamma_{0}$ (in $s^{-1}$ ) is the inverse photon life time. The feedback rate $\gamma$ is defined as

$$
\gamma=\frac{\left(1-r_{1}^{2}\right) r_{2}}{r_{1} \tau_{i n}}
$$

In this equation, $r_{1}\left(r_{1}=\sqrt{R_{1}}\right)$ and $r_{2}\left(r_{2}=\sqrt{R_{2}}\right)$ are the reflection coefficients of the output facet of the laser and of the external mirror, respectively. In these notations, the relaxation oscillation (RO) frequency $\omega_{R}$ and the damping rate $\Gamma_{R}$ of the solitary laser are defined by

$$
\omega_{R}=\sqrt{\Gamma_{0} \xi} \quad \text { and } \quad \Gamma_{R}=\frac{1}{2}\left(\frac{1}{T_{1}}+\xi I\right)
$$

where $I$ [dimensionless, $I=\left(J-J_{t h}\right) / \Gamma_{0}$ ] is the photon number for the solitary laser.

It would be more convenient to use the dimensionless form of the above equations. Defining 


$$
N=\frac{\xi n}{2 \Gamma_{0}}, \quad s=\Gamma_{0} t \quad \text { and } \quad Y=E \sqrt{\frac{\xi T_{1}}{2}}
$$

then Equations (1) and (2) become

$$
\begin{gathered}
\dot{Y}(s)=(1+j \alpha) N(s) Y(s)+\eta Y(s-\theta) \exp \left(-j \Delta_{0}\right) \\
\dot{N}(s) T=P-N(s)-[1+2 N(s)]|Y(s)|^{2}
\end{gathered}
$$

where

$$
\eta=\frac{\gamma}{\Gamma_{0}}, \quad \theta=\Gamma_{0} \tau, \quad \Delta_{0}=\omega_{0} \tau=\Omega_{0} \theta, \quad T=\Gamma_{0} T_{1} \quad \text { and } \quad P=\frac{\xi}{2} T_{1} \frac{J-J_{\text {th }}}{\Gamma_{0}}
$$

In (8), $\eta$ is the normalized feedback rate, $\Delta_{0}$ is the initial feedback phase and $P$ is the controlling parameter, called the pump parameter above threshold.

\subsection{Stability analysis}

The L-K equations (6) and (7) describe the dynamic response of a single-mode laser subject to optical feedback from a distant mirror. By using $Y=\operatorname{Rexp}(j \Phi)$ as the expression for the electrical field, we obtain $(Z=N)$

$$
\begin{gathered}
\dot{R}(s)=Z(s) R(s)+\eta R(s-\theta) \cos \left[\Phi(s-\theta)-\Phi(s)-\Delta_{0}\right] \\
\dot{\Phi}(s)=\alpha Z(s)+\eta \frac{R(s-\theta)}{R(s)} \sin \left[\Phi(s-\theta)-\Phi(s)-\Delta_{0}\right] \\
\dot{Z}(s) T=P-Z(s)-[1+2 Z(s)] R^{2}(s)
\end{gathered}
$$

Equations (9)-(11) admit a basic steady-state solution

$$
\ddot{Y}=\ddot{R} \exp (j \tilde{\Phi}) \text { and } \ddot{Z}
$$

where $\tilde{R}, \tilde{\Phi}$ and $\tilde{Z}$ are given by

$$
\tilde{R}=A=\sqrt{\frac{P+\eta \cos (\Delta)}{1-2 \eta \cos (\Delta)}}, \tilde{\Phi}=Q(s)=\left(\Omega-\Omega_{0}\right) s \text { and } \tilde{Z}=B=-\eta \cos (\Delta)
$$

$\tilde{Y}$ (together with $\tilde{Z}$ ) is called an external-cavity mode or ECM. The feedback phase $\Delta($ $\Delta=\omega \tau=\Omega \theta)$ associated with this mode must be a solution of the following phase equation

$$
\Delta-\Delta_{0}=-\eta \theta[\alpha \cos (\Delta)+\sin (\Delta)]
$$

We investigate the linear stability properties of Eqs. (9)-(11) by introducing the small perturbations $u, v$ and $w$. By inserting $R=A+u, \Phi=Q+v$ and $Z=B+w$ into these equations, we obtain the following linearzed equations for $u, v$ and $w$ 


$$
\begin{gathered}
\dot{u}(s)=-B[u(s-\theta)-u(s)]+\eta A \sin (\Delta)[v(s-\theta)-v(s)]+A w(s) \\
\dot{v}(s)=\frac{-\eta \sin (\Delta)}{A}[u(s-\theta)-u(s)]-B[v(s-\theta)-v(s)]+\alpha w(s) \\
\dot{w}(s) T=-2 A(1+2 B) u(s)-\left(1+2 A^{2}\right) w(s)
\end{gathered}
$$

We solve these equations by looking for a solution of the form $u(s)=a \exp (\lambda s)$, $v(s)=b \exp (\lambda s)$ and $w(s)=c \exp (\lambda s)$. We then obtain the following problem for the coefficients $a, b$ and $c$

$$
\lambda\left(\begin{array}{l}
a \\
b \\
c
\end{array}\right)=L_{J}\left(\begin{array}{l}
a \\
b \\
c
\end{array}\right) \text { or } \lambda V_{J}=L_{J} V_{J}
$$

where $V_{J}$ is the eigenvector of the Jacobian matrix $L_{J}$ given by

$$
L_{J}=\left(\begin{array}{ccc}
-B F & \eta F A \sin (\Delta) & A \\
-\eta F A^{-1} \sin (\Delta) & -B F & \alpha \\
-2 A \varepsilon(1+2 B) & 0 & -\varepsilon\left(1+2 A^{2}\right)
\end{array}\right)
$$

with $F=\exp (-\lambda \theta)-1$ and $\varepsilon=T^{-1}$. A nontrivial solution is possible only if the growth rate $\lambda$ satisfies the condition $\operatorname{det}\left(L_{I}-\lambda I_{3}\right)=0$ (with $I_{3}$ : identity matrix), leading to a characteristic equation for $\lambda\left(\lambda=\lambda_{1}+j \lambda_{2}\right)$. Under the approximation of low feedback rate (i.e. small values of $\gamma \tau$ ), there is only one ECM. By applying an asymptotic analysis to (13) and to the characteristic equation [details in (Erneux, 2000, 2008)], we obtain the normalized damping rate $\lambda_{1}$ and $\mathrm{RO}$ frequency $\lambda_{2}$

$$
\begin{aligned}
& \lambda_{1}=\frac{-\Gamma_{R}}{\Gamma_{0}}-\frac{\gamma}{\Gamma_{0}} \sin ^{2}\left(\frac{\omega_{R} \tau}{2}\right) \sqrt{1+\alpha^{2}} \cos \left(\Delta_{0}-\psi\right) \\
& \lambda_{2}= \pm \frac{\omega_{R}}{\Gamma_{0}} \mp \frac{\gamma}{2 \Gamma_{0}} \sin \left(\omega_{R} \tau\right) \sqrt{1+\alpha^{2}} \cos \left(\Delta_{0}-\psi\right)
\end{aligned}
$$

where $\psi=\operatorname{arctg}(\alpha)$. Equation (21) implies, among other things, that if $\omega_{R} \tau$ is an odd multiple of $\pi$, the feedback will not affect the RO frequency of the solitary laser.

\section{Further discussions}

\subsection{First Hopf bifurcation}

The stability of an equilibrium (or a fixed) point $\tilde{Y}$ of the electrical field $Y$ is determined by the eigenvalue $\lambda$ of the Jacobian matrix $L_{J}$. With increasing feedback rate, an ECM will experience a change of its stability through a Hopf bifurcation. Under the assumption of low feedback rate, this mode will become unstable if $\lambda_{1}$ changes from negative to positive 
values, corresponding to an undamped optical power proportional to $|Y|^{2}$. As can be seen from Eq.(20), such a situation occurs only if

$$
\cos \left(\Delta_{0}-\psi\right)<0
$$

It follows that an ECM will lose its stability at $\lambda_{1}=0$. For a real laser system, this threshold is commonly called the onset of "coherence collapse», since the laser exhibits a drastic linewidth broadening in this state. From Eq.(20) and (8), the normalized critical feedback rate is given by

$$
\eta_{c}=\frac{-\Gamma}{\sin ^{2}\left(\frac{\omega_{R} \tau}{2}\right) \sqrt{1+\alpha^{2}} \cos \left(\Delta_{0}-\psi\right)}
$$

where $\Gamma=\Gamma_{R} / \Gamma_{0}$. It would be worthwhile to notice here that although the term $\cos \left(\Delta_{0}-\psi\right)$ should be negative $(\eta \geq 0)$, it is not necessarily equal to -1 .

\subsection{Stability characterization of an ECM}

The stability of an ECM will be completely determined if the three components $a, b$ and $c$ of the eigenvector $V_{J}$ are known. They can be derived from Eqs.(18) and (19) and written as functions of $a$. Since the value of $a$ can arbitrarily be chosen $(a \neq 0)$, we thus take $a=1$ and express these coefficients as follows

$$
a=1, \quad b=(k+h \alpha) /(\lambda+B F) \quad \text { and } \quad c=h
$$

where

$$
k=\frac{-\eta \sin (\Delta)}{A}, \quad f=\frac{-2 A \varepsilon(1+2 B)}{\lambda}, \quad g=\frac{\varepsilon\left(1+2 A^{2}\right)}{\lambda} \quad \text { and } h=\frac{f}{1+g}
$$

In this way, the time-dependent electrical field of an ECM is determined by

$$
R=A+\exp (\lambda s), \quad \Phi=Q+b \exp (\lambda s) \text { and } Z=B+c \exp (\lambda s)
$$

Phase portraits in the different state spaces have been proposed to illustrate the stability properties of an ECM, as for example in the equivalent planes of $(I, n)$ (Mørk et al., 1988) and of $\left(\Phi(s)-\Phi(s-\theta),-\eta \cos \left[\Phi(s)-\Phi(s-\theta)+\Delta_{0}\right]\right)$ (Sano, 1994), and also in the planes of the different components of the electrical field and the carrier density (Tromborg \& Mørk, 1990). Here we simply illustrate the transient trajectories (for $s=0-10000$ ), in the complex plane, of $R, \Phi$ and $Z$ (Figs. 2-5), for a composite laser system with the normalized feedback rate around its instability threshold. The laser parameters and their values are given in Table 1. As can be seen from these figures, equivalent to the above mentioned methods, such a representation allows a straightforward interpretation of the transient behaviors of a composite laser system. 


\begin{tabular}{|c|c|l|}
\hline symbol & value & \multicolumn{1}{c|}{ description } \\
\hline$\alpha$ & 5 & linewidth enhancement factor \\
\hline$\xi$ & $2 \times 10^{-4} \mathrm{~s}^{-1}$ & differential gain \\
\hline$\Gamma_{0}$ & $4 \times 10^{11} \mathrm{~s}^{-1}$ & inverse photon life time \\
\hline$T_{1}$ & $14 \times 10^{-10} \mathrm{~s}$ & carrier life time \\
\hline$\lambda_{0}$ & $1.55 \times 10^{-6} \mathrm{~m}$ & solitary-laser wavelength \\
\hline$L$ & $0.03 \mathrm{~m}$ & external-cavity length \\
\hline$\Delta_{0}$ & $-2.02683397 \mathrm{rad}$ & initial feedback phase \\
\hline$\Delta$ & $-1.54276496 \mathrm{rad}$ & ECM feedback phase \\
\hline$P$ & $5 \times 10^{-3}$ & pump parameter above threshold \\
\hline
\end{tabular}

Table 1. Laser parameters and their values used in most of the figures in the text.

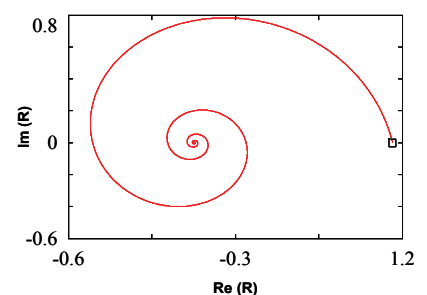

(a)

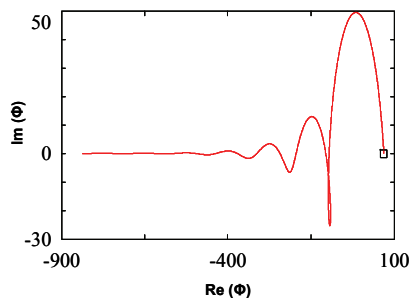

(b)

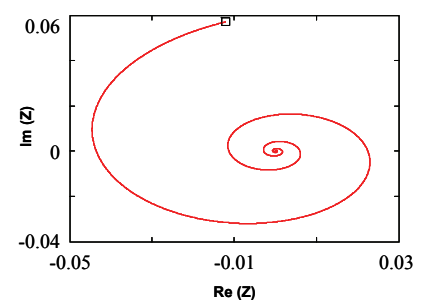

(c)

Fig. 2. Stability features of a solitary laser $(\eta=0)$. Phase portraits in the complex plane for the electrical field $Y$ (attractor solution). Trajectories for (a): the amplitude $R$, (b): the phase $\Phi$ and (c): the carrier density $Z$. The square symbol denotes the initial state at $s=0$.

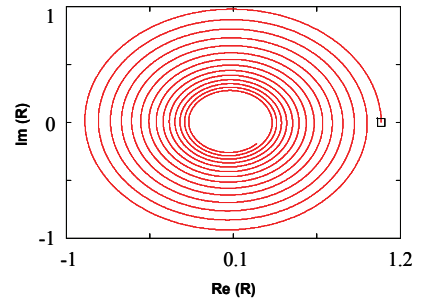

(a)

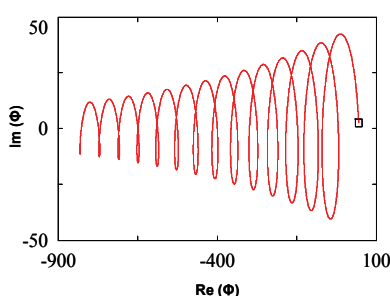

(b)

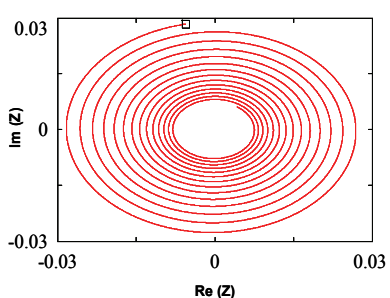

(c)

Fig. 3. Stability features of the same laser with the normalized feedback rate close to its threshold $\left(\eta=0.85 \eta_{c}\right)$. Phase portraits in the complex plane for the electrical field $Y$ (attractor solution). Trajectories for (a): the amplitude $R$, (b): the phase $\Phi$ and (c): the carrier density $Z$. The square symbol denotes the initial state at $s=0$. 


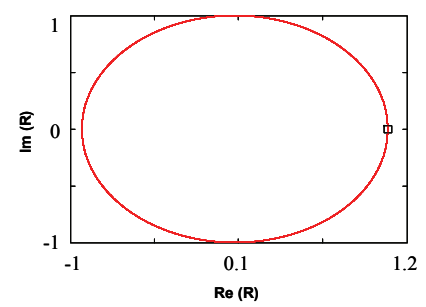

(a)

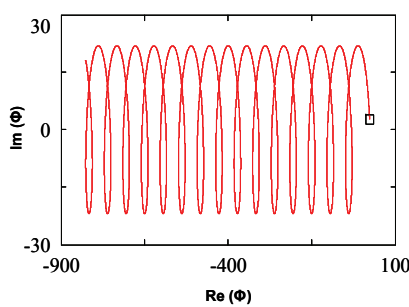

(b)

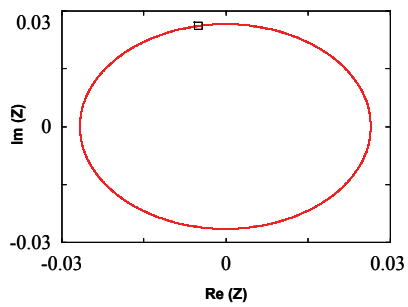

(c)

Fig. 4. Stability features of the same laser at the onset of coherence collapse $\left(\eta=\eta_{c}\right)$. Phase portraits in the complex plane for the electrical field $Y$ (limit-cycle solution). Trajectories for (a): the amplitude $R,(b)$ : the phase $\Phi$ and (c): the carrier density $Z$. The square symbol denotes the initial state at $s=0$.

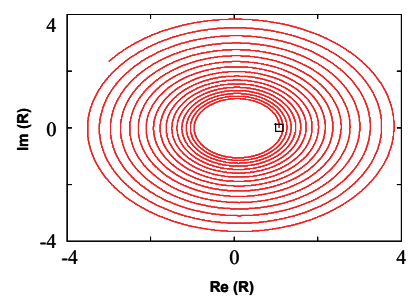

(a)

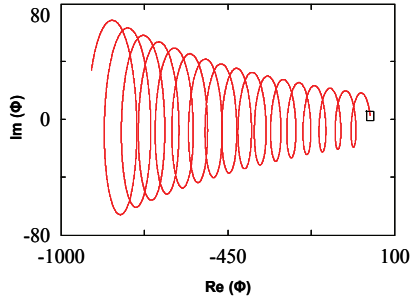

(b)

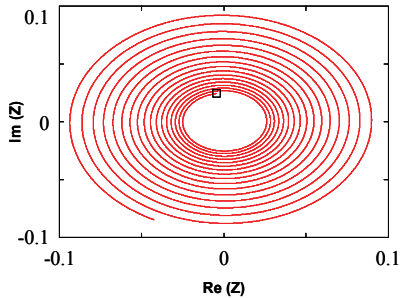

(c)

Fig. 5. Stability features of the same laser becoming now coherence-collapsed $\left(\eta=1.15 \eta_{c}\right)$. Phase portraits in the complex plane for the electrical field $Y$ (repellor solution). Trajectories for (a): the amplitude $R$, (b): the phase $\Phi$ and (c): the carrier density $Z$. The square symbol denotes the initial state at $s=0$.

\section{Qualitative interpretation}

As mentioned in the introduction, a preliminary interpretation of the experimental results obtained with InAs/InP QDash F-P lasers will be performed in this section using the analytical expressions derived in Section 3. We concentrate on two laser states in which some typical phenomena were observed in these experiments: the onset of coherence collapse (CC) and the regime of fully-developed coherence collapse.

\subsection{Onset of coherence collapse}

This state corresponds to the first Hopf bifurcation of the L-K rate equations. We hence have for the critical feedback rate

$$
\gamma_{c}=\frac{-\Gamma_{R}}{\sin ^{2}\left(\frac{\omega_{R} \tau}{2}\right) \sqrt{1+\alpha^{2}} \cos \left(\Delta_{0}-\psi\right)}
$$

If the feedback level is defined as the ratio of the feedback power to the solitary-laser emission power, it can be written at the CC onset as 


$$
f_{c}=\frac{P_{1}}{P_{0}}=\frac{\left|\left(1-r_{1}^{2}\right) r_{2} E(t-\tau) \exp \left(-j \omega_{0} \tau\right)\right|^{2}}{|E(t)|^{2}}
$$

In steady state, we have from Eq.(3)

$$
f_{c}=\left(1-r_{1}^{2}\right)^{2} r_{2}^{2}=\gamma_{c}^{2} r_{1}^{2} \tau_{i n}^{2}=\frac{\Gamma_{R}^{2} \tau_{i n}^{2} R_{1}}{\sin ^{4}\left(\frac{\omega_{R} \tau}{2}\right)\left(1+\alpha^{2}\right) \cos ^{2}\left(\Delta_{0}-\psi\right)}
$$

In general, the critical feedback level $f_{c}$ is a parabolic function of the pumping current above threshold

$$
f_{c}=\frac{\tau_{i n}^{2} R_{1}}{4 T_{1}^{2} \sin ^{4}\left(\frac{\omega_{R} \tau}{2}\right)\left(1+\alpha^{2}\right) \cos ^{2}\left(\Delta_{0}-\psi\right)}\left[1+2 \xi T_{1} \Gamma_{0}^{-1}\left(J-J_{t h}\right)+\xi^{2} T_{1}^{2} \Gamma_{0}^{-2}\left(J-J_{t h}\right)^{2}\right]
$$

It can easily be shown that the quadratic dependence of $f_{c}$ on $J-J_{\text {th }}$ can be neglected if the RO frequency $\omega_{R}$ satisfies $\omega_{R}<<\sqrt{2 /\left(T_{1} \Gamma_{0}^{-1}\right)}$. For example, if the photon lifetime $\Gamma_{0}^{-1}$ and the carrier lifetime $T_{1}$ are taken respectively as $2.5 \mathrm{ps}$ and $1.4 \mathrm{~ns}$ (Table 1), we have $\omega_{R} /(2 \pi)<<3.8 \mathrm{GHz}$. In this case, Eq.(30) is simplified as

$$
f_{c}=\frac{\tau_{i n}^{2} R_{1}}{4 T_{1}^{2} \sin ^{4}\left(\frac{\omega_{R} \tau}{2}\right)\left(1+\alpha^{2}\right) \cos ^{2}\left(\Delta_{0}-\psi\right)}\left[1+2 \xi T_{1} \Gamma_{0}^{-1}\left(J-J_{t h}\right)\right]
$$

where the slope is proportional to the differential gain $\xi$ and inversely proportional to the LEF $\alpha$. The experimental evidence of the impact of $\alpha$ and $\xi$ on $f_{c}$ for InAs/InP QDash F$P$ lasers is reported in (Azouigui et al., 2008). In this study, the linear dependence of $f_{c}$ on $J-J_{\text {th }}$ was systematically observed with these lasers, which were fabricated on the basis of three different material (dash-in-a-barrier, dash-in-a-well and tunnel-injection) structures and processed from several structures exhibiting various values of $\alpha$ and $\xi$ (Fig. 6).

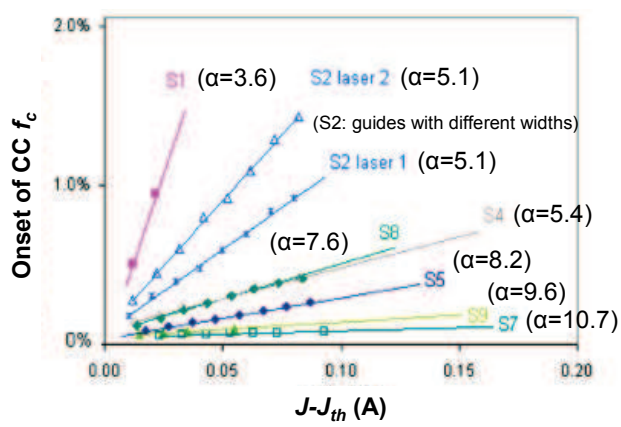

Fig. 6. Onsets of CC measured as a function of the pumping current above threshold, for seven laser structures, using an $18 \mathrm{~m}$-long optical-fiber-based external cavity (Azouigui et al., 2008). All the devices are 600- $\mu m$-long as-cleaved F-P lasers. The most resistant lasers were fabricated from the structure $S_{1}$, since it has the lowest $\alpha$ value as well as the highest $\xi$ one. 


\subsection{Fully-developed coherence collapse}

It is widely-recognized that when a laser, subject to moderate amounts of optical feedback, operates close to its solitary threshold, it exhibits a rather complex behavior due to the socalled low-frequency fluctuations (LFF), and that for a large enough pumping current there will occur a transition from the regime of LFF to the regime of fully-developed coherence collapse (FDCC). In a recent experimental investigation, the LFF regime was observed around laser threshold $(13 \mathrm{~mA})$ at $-1.2 \mathrm{~dB}$ feedback, for a $600-\mu m$-long InAs/InP QDash F-P laser emitting at $1.57 \mu \mathrm{m}$ and using a free-space setup with a short external cavity $(L=0.5 \mathrm{~m})$ (Azouigui et al., 2007b). In these experiments, the regime of FDCC was attained at about $30 \mathrm{~mA}$. A number of feedback-induced phenomena were manifested in the frequency domain, such as RF (radio-frequency) noise enhancement, frequency shift and peak broadening (Fig. 7). In this section, we will give some explanations of all these phenomena by using the formalism developed in Section 3. An intensive discussion about the physical origin of the LFF regime and the mechanism of its transition to the FDCC regime, based on numerical simulations of the L-K rate equations, is found in (Sano, 1994) and (Zamora-Munt et al., 2010).

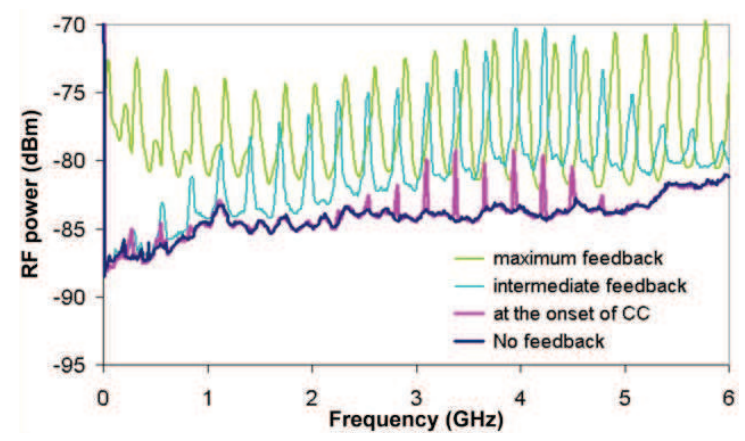

Fig. 7. Measured RF spectra with increasing feedback level, for a pumping current of $30 \mathrm{~mA}$ (Azouigui et al., 2007b).

Let us first have a look at the expression for the intensity of an ECM. It can be derived from (26) and be written in general as

$$
\begin{aligned}
& \begin{aligned}
|Y(s)|^{2} & =\exp \left(-2 \Phi_{2}\right)|R(s)|^{2} \\
& =\exp \left(-2 \Phi_{2}\right)\left[A^{2}+\exp \left(2 \lambda_{1} s\right)+2 A \exp \left(\lambda_{1} s\right) \cos \left(\lambda_{2} s\right)\right]
\end{aligned} \\
& \text { with: } \\
& \Phi_{2}=\exp \left(\lambda_{1} s\right)\left[b_{1} \sin \left(\lambda_{2} s\right)+b_{2} \cos \left(\lambda_{2} s\right)\right]
\end{aligned}
$$

In this equation, $\Phi_{2}$ is the imaginary part of $\Phi$, which is non-null in steady state for a coherence-collapsed laser; $b_{1}$ and $b_{2}$ are respectively the real and imaginary parts of $b$. At the onset of $\operatorname{CC}\left(\lambda_{1}=0\right)$, this equation is reduced to

$$
|Y(s)|_{c}^{2}=\exp \left\{-2\left[b_{1} \sin \left(\lambda_{2} s\right)+b_{2} \cos \left(\lambda_{2} s\right)\right]\right\}\left[A^{2}+1+2 A \cos \left(\lambda_{2} s\right)\right]
$$


In this particular case, the $\lambda_{1}$-dependent exponential terms disappear and the intensity of an ECM displays equal-amplitude pulses. As an example, the intensity distributions of an ECM for the normalized feedback rate around its instability threshold, calculated from Eq.(32), are illustrated in Fig. 8. In this example, the laser is pumped by a relatively strong current $\left(P=5 \times 10^{-3}\right)$. This, as expected, results in a rapid damping (when $\eta<\eta_{c}$ ) of its emission power to a steady-state value proportional to $A^{2}$, which is close to zero according to our notations [as can be seen in (13) for the definition of $A$ and in (5) where $|E|^{2} \propto 10^{14}|Y|^{2}$ ].

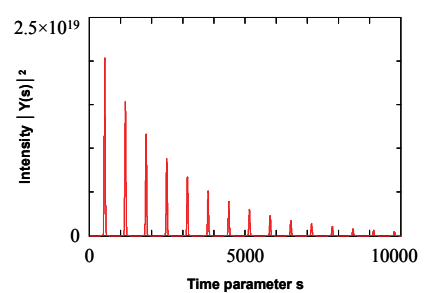

(a)

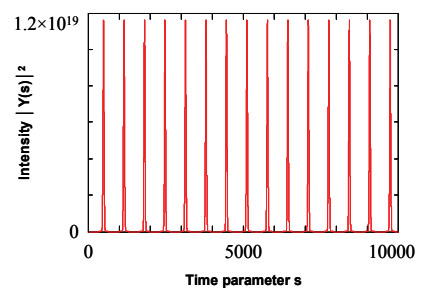

(b)

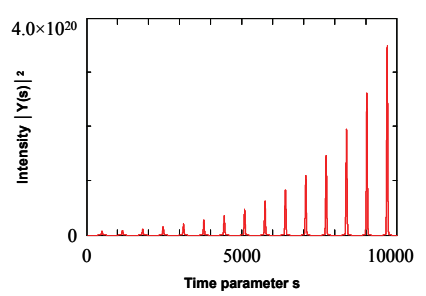

(c)

Fig. 8. Time traces of the transient intensity $|Y(s)|^{2}$ for the normalized feedback rate around its critical threshold. (a) $\eta=0.99 \eta_{c}$; (b) $\eta=\eta_{c}$; (c) $\eta=1.01 \eta_{c}$.

\subsubsection{Feedback-enhanced RF noise}

The intensity distributions of an ECM are plotted in Fig. 9 (a), for two values of the normalized feedback rate and for a great $P$ value $\left(P=5 \times 10^{-3}\right)$. This result suggests that for a coherence-collapsed laser pumped by a large current, a small excess (a few percentages) in feedback level could lead to a significant increase of the RF noise, and therefore of the spectrum power as shown in Fig. 7.

\subsubsection{Feedback-induced frequency shift and peak broadening}

Figure 7 also shows that in the frequency domain, the increase of the RF noise with the feedback level is accompanied by a shift, to the greater frequencies (blue shift), of the peaks and their broadening. According to Eq.(32), at the CC onset as well as inside the CC regime, the undamped optical power of an ECM will oscillate at the frequency $\lambda_{2}$ (see also Fig. 8), which is greater than the normalized solitary-laser RO frequency $\omega_{R} / \Gamma_{0}$ if $\omega_{R} \tau<\pi \bmod 2 \pi$ [ $\sin \left(\omega_{R} \tau\right)>0$ ]. This equation also predicts a peak broadening with the feedback level. Since $\lambda_{1} \geq 0$ for a coherence-collapsed laser, these peaks will have the narrowest width at the onset of CC. Figure 9 (b) depicts the calculated Fourier spectra of an ECM, showing a good qualitative agreement with the experimental findings illustrated in Fig. 7.

\subsubsection{Current-induced frequency increasing and peak broadening}

In the case of a given feedback rate and for a short external cavity $\left(\omega_{R} \tau<\pi / 2\right)$, Equation (21) suggests a monotonous increasing, through the term $\sin \left(\omega_{R} \tau\right)$, of the RO frequency $\lambda_{2}$ with the pumping current $J$ when the laser system passes from LFF to FDCC regime [Fig. 10, (a)]. The nonlinear relation between $\lambda_{2}$ and $J$ can be checked by inspection of Fig. 10, (b), where the RF spectra were obtained with a $0.5 \mathrm{~m}$-long external cavity. 


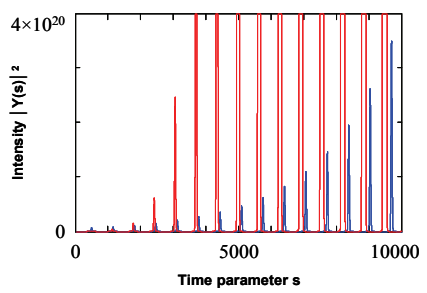

(a)

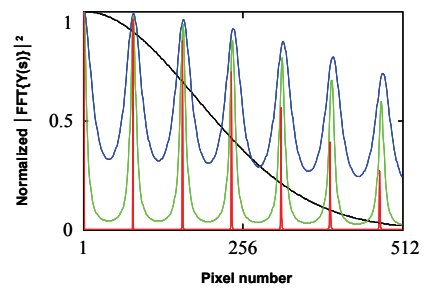

(b)

Fig. 9. Feedback-induced phenomena inside the FDCC regime with $P=5 \times 10^{-3}$, predicted by Eq.(32). The other parameter values are given in Table 1. (a) Time traces of the feedbackenhanced RF noise in terms of ECM intensity. Blue: $\eta=1.01 \eta_{c}$; Red: $\eta=1.05 \eta_{c}$. (b)

Feedback-enhanced frequency blue shift and peak broadening (FFT: Fast Fourier Transform). Black: $\eta=0$; Red: $\eta=\eta_{c}$; Green: $\eta=1.01 \eta_{c}$; Blue: $\eta=1.02 \eta_{c}$. The first pixel corresponds to the position of the normalized solitary-laser RO frequency $\omega_{R} / \Gamma_{0}$.

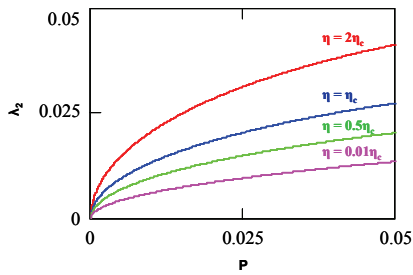

(a)

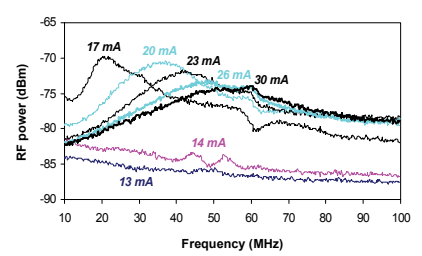

(b)

Fig. 10. Current-induced frequency increasing and peak broadening during the transition from the LFF regime to the FDCC regime. (a) Variation of $\lambda_{2}$ as a function of $P$ for several values of the normalized feedback rate, calculated from Eq.(21). (b) Measured RF spectra at $-1.2 d B$ feedback (Azouigui et al., 2007b). The transition period corresponds roughly to a variation of the pumping current from $17 \mathrm{~mA}$ to $30 \mathrm{~mA}$.

\section{Comparison with a classical model}

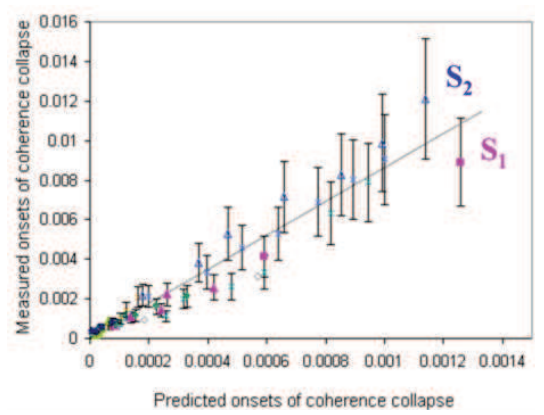

Fig. 11. Measured values of the CC onset versus predicted ones deduced from Eq.(10) in (Helms \& Petermann, 1990) (Azouigui et al., 2008). Compared to the structure $S_{2}$ (see Fig. 6), the structure $S_{1}$ has a smaller slope, which means that its measured onset values are closer to the predicted ones. 
In this section, the difference between Eq.(29) and the model proposed by Helms and Petermann (denoted as the H-P model, Helms \& Petermann, 1990) will be discussed from a practical point of view, for the case of long external cavities (where $\omega_{R} \tau$ is set to be an odd multiple of $\pi$ ). A deeper analysis of the cause for this difference is beyond the scope of the present work. We are motivated by such an approach also because when we undertook an interpretation of Fig. 6, we found that although for all structures the threshold values predicted by the H-P model follow the same trend as the measured ones, a difference of $8-10 \mathrm{~dB}$ is systematically obtained between the measured and predicted values (Fig. 11).

Equation (29) predicts a linear dependence of the onset of CC on the output-facet reflectivity of the laser diode. By assuming a long external cavity, it is simplified as

$$
f_{c}=\frac{\Gamma_{R}^{2} \tau_{i n}^{2} R_{1}}{\left(1+\alpha^{2}\right) \cos ^{2}\left(\Delta_{0}-\psi\right)}
$$

Remember that $\cos \left(\Delta_{0}-\psi\right)$ should take negative values. The critical feedback level predicted by the H-P model, which was established in the small-signal domain with the help of a transfer function from the modulation current to the modulated optical power, is determined by [Eq.(8) in Helms \& Petermann, 1990]

$$
f_{c}^{H-P}=\frac{\Gamma_{R}^{2} \tau_{i n}^{2}}{\left(1+\alpha^{2}\right) 16 C_{l}^{2}}
$$

where $C_{l}$ denotes the coupling strength from the laser cavity to the external cavity. It is given by $C_{l}=\left(1-R_{1}\right) /\left(2 \sqrt{R_{1}}\right)\left(0 \leq C_{l} \leq \infty\right)$ for a F-P laser. The difference between these two models is expressed by $\beta$, defined as the ratio of $f_{c}^{H-P}$ and $f_{c}$ in logarithmic form

$$
\beta(d B)=10 \log _{10} \frac{f_{c}^{H-P}}{f_{c}}=10 \log _{10} \frac{\cos ^{2}\left(\Delta_{0}-\psi\right)}{4\left(1-R_{1}\right)^{2}}=10 \log _{10}\left[\cos ^{2}\left(\Delta_{0}-\psi\right)\right]-2.9
$$

where we have taken $R_{1}=0.3$ for an as-cleaved F-P laser. As indicated in this expression, $\beta$ will be mainly affected by the term $\Delta_{0}-\psi$. This term, varying in the range $\pi / 2 \bmod 2 \pi<\Delta_{0}-\psi \leq \pi \bmod 2 \pi$, corresponds to a very large variation range of $\beta$ [Fig. 12, (a)]. For example, if $\Delta_{0}-\psi=\pi$, we have $\cos \left(\Delta_{0}-\psi\right)=-1$ and $\beta=-2.9 d B$. One radian less for the initial feedback phase, i.e. $\Delta_{0}-\psi=\pi-1$, will result in $\cos \left(\Delta_{0}-\psi\right)=-0.54$ and $\beta=-8.3 d B$. The absence of the cosine term in the denominator of $f_{c}^{H-P}$ makes the H-P model correspond to the most unfavorable situation. For a given initial frequency $\omega_{0}$, two factors can considerably influence the $\beta$ ratio: the external delay time $\tau$ and the LEF $\alpha$. Singularity occurs if the initial feedback phase $\Delta_{0}$ approaches $\psi+(\pi / 2 \bmod 2 \pi)$. Moreover, $\beta$ is highly sensitive to very small variations of the externalcavity length $L$, because of the ratio $L / \lambda_{0}$ in the expression of $\Delta_{0}$, where $L$ is in meter and $\lambda_{0}$ in micron. An example is given in Fig. 12, (b).

We can see from this specific case that for a given $\alpha$ value [ $\alpha$ should be greater than 1 , in order that Eq.(35) holds, according to the H-P model], the values of $|\beta|$ can be quite different due to small oscillations of the $L$ value, and that for a positive (negative) increment of $L$, 
$|\beta|$ increases (decreases) with $\alpha$. A further analysis of this figure would give some qualitative explications of the observations stated above (Fig. 11). (1) For the curve where $L$ is taken exactly as $18 \mathrm{~m},|\beta|$ increases with $\alpha$. This means that a laser with a smaller $\alpha$ value would have a CC onset value closer to that predicted by the H-P model. Experimentally, we did observe such behavior. As shown in Fig. 11, the structure $S_{1}$ $(\alpha=3.6)$ has a smaller $|\beta|$ ratio than the structure $S_{2} \quad(\alpha=5.1)$; (2) The incertitude of the measurement (within hundreds of microns) of the external-cavity length can affect considerably the $\beta$ value.

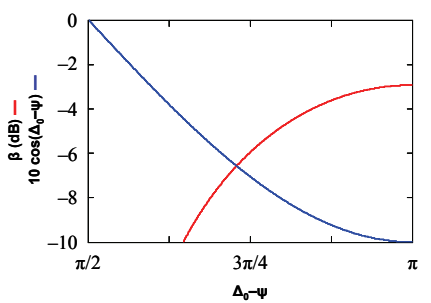

(a)

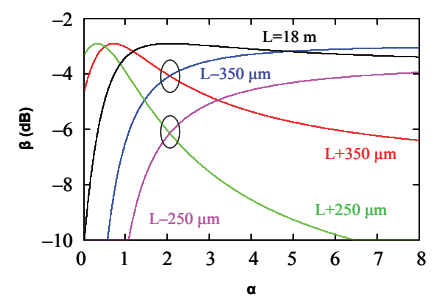

(b)

Fig. 12. (a) Dependence of $\beta$ on $\Delta_{0}-\psi$ (red curve), in the range $\pi / 2<\Delta_{0}-\psi \leq \pi$. For values of $\Delta_{0}-\psi$ away from $\pi$, there exists a great difference between Eq.(34) and Eq.(35). (b) Variation of $\beta$ as a function of $\alpha$, for an $18 m$-long optical-fiber-based external cavity. The refractive index of the fiber was taken as 1.5 for a solitary laser emitting at the wavelength of $1.55 \mu \mathrm{m}$. The small increments around $L$ were chosen arbitrarily.

\section{Conclusion}

The asymptotic approach provides a useful tool for stability modeling of bulk, quantumwell as well as quantum-dash semiconductor lasers with optical feedback. The analytical solution of the Lang and Kobayashi rate equations derived using this approach enables a complete description, in the phase space and also in the time domain, of an external-cavity mode for a composite laser system close to, at the onset of as well as inside the coherencecollapse regime. More specifically, by use of this model, the temporal and spectral behaviors of the laser system in the regime of fully-developed coherence collapse, usually numerically analyzed, can be described in an analytical way. This model gives a quite good physical insight of the phenomena recently observed with 1.55- $\mu \mathrm{m}$ InAs/InP quantum-dash FabryPerot lasers, when these lasers were characterized for their resistivity to optical feedback originating from two different external-cavity configurations: one was based on a $0.5 \mathrm{~m}$ long free-space setup and another was composed of an $18 \mathrm{~m}$-long optical fiber. Compared to the H-P model, the expression for determination of the threshold of coherence collapse derived in Section 4, namely Eq.(29), is found to be in better agreement (a «gain» of at least $\sim 3 \mathrm{~dB}$ ) with the experimental data obtained with these lasers fabricated from quantumdash-based nanostructures. However, it seems that its use would require, for each value of the pumping current, more certitude on the measurement of the solitary-laser wavelength and also on that of the external-cavity length.

The dynamics of the low-frequency fluctuations will be investigated in the near future using this approach in comparison with numerical approaches so far reported in the literature. 


\section{Acknowledgments}

One of the authors (Q. Zou) would like to acknowledge K. Merghem, A. Martinez, and A. Ramdane (CNRS, Laboratory for Photonics and Nanostructures, France), and A. Accard, N. Chimot, J.-G. Provost, and F. Lelarge (Alcatel-Thales III-V Lab, a joint laboratory of AlcatelLucent Bell Labs and Thales Research \& Technology, France) for fruitful collaborations. He would also like to thank T. Erneux (Université Libre de Bruxelles, Unité Optique NonLinéaire Théorique, Belgium) for helpful discussions.

\section{References}

Azouigui, S.; Dagens, B.; Lelarge, F.; Provost, J.G.; Accard, A.; Grillot, F.; Martinez, A.; Zou, Q. \& Ramdane, A. (2007). Tolerance to Optical Feedback of 10 Gbps QuantumDash-Based Lasers Emitting at $1.51 \mu \mathrm{m}$. IEEE Photonics Technology Letters, Vol.19, pp. 1181-1183

Azouigui, S.; Kelleher, B.; Hegarty, S.P.; Huyet, G.; Dagens, B.; Lelarge, F.; Accard, A.; Make, D.; Le Gouezigou, O.; Merghem, K.; Martinez, A.; Zou, Q. \& Ramdane, A. (2007). Coherence Collapse and Low-Frequency Fluctuations in Quantum-Dash Based Lasers Emitting at $1.57 \mu \mathrm{m}$. Optics Express, Vol.15, pp. 14155-14162

Azouigui, S.; Dagens, B.; Lelarge, F.; Accard, A.; Make, D.; Le Gouezigou, O.; Merghem, K.; Martinez, A.; Zou, Q. \& Ramdane, A. (2008). Systematic Investigation of InAs/InP Quantum-Dash Based Lasers under External Optical Feedback. Appl. Phys. Lett., Vol.92, 201106

Azouigui, S.; Dagens, B.; Lelarge, F.; Provost, J.G.; Make, D.; Le Gouezigou, O.; Accard, A.; Martinez, A.; Merghem, K.; Grillot, F.; Dehaese, O.; Piron, R.; Loualiche, S.; Zou, Q. \& Ramdane, A. (2009). Optical Feedback Tolerance of Quantum-Dot- and Quantum-Dash-Based Semiconductor Lasers Operating at $1.55 \mu \mathrm{m}$. IEEE Journal of Selected Topics in Quantum Electronics, Vol.15, pp. 764-773

Bimberg, D.; Grundmann, M. \& Ledentsov, N.N. (1999). Quantum Dot Heterostructures (Wiley, New York).

Binder, J.O. \& Cormack, G.D. (1989). Mode Selection and Stability of a Semiconductor Laser with Weak Optical Feedback. IEEE Journal of Quantum Electronics, Vol.35, pp. 22552259

Erneux, T. (2000). Asymptotic Methods Applied to Semiconductor Laser Models. SPIE 3944, Physics \& Simulations of Optoelectronic Devices VIII

Erneux, T. (2008). First Hopf Bifurcation of the Lang-Kobayashi Equations. Private communication

Grillot, F.; Thedrez, B.; Gauthier-Lafaye, O.; Martineau, M.F.; Voiriot, V.; Lafragette, J.L.; Gentner, J.L. \& Silvestre, L. (2003). Coherence Collapse Threshold of $1.3 \mu \mathrm{m}$ Semiconductor DFB Lasers. IEEE Photonics Technology Letters, Vol.15, pp. 9-11

Grillot, F.; Thedrez, B. \& Duan, G.H. (2004). Feedback Sensitivity and Coherence Collapse Threshold of Semiconductor DFB Lasers with Complex Structures. IEEE Journal of Quantum Electronics, Vol.40, pp. 231-240

Helms, J. \& Petermann, K. (1990). A Simple Analytic Expression for the Stable Operation Range of Laser Diodes with Optical Feedback. IEEE Journal of Quantum Electronics, Vol.26, No.5, pp. 833-836 
Lang, R. \& Kobayashi, K. (1980). External Optical Feedback Effects on Semiconductor Injection Laser Properties. IEEE Journal of Quantum Electronics, Vol.QE-16, No.3, pp. 347-355

Lelarge, F.; Dagens, B.; Renaudier, J.; Brenot, R.; Accard, A.; Van Dijk, F.; Make, D.; Le Gouezigou, O.; Provost, J.G.; Poingt, F.; Landreau, J.; Drisse, O.; Derouin, E.; Rousseau, B.; Pommereau, F. \& Duan, G.H. (2007). Recent Advances on InAs/InP Quantum Dash Based Semiconductor Lasers and Optical Amplifiers Operating at $1.55 \mu \mathrm{m}$. IEEE Journal of Selected Topics in Quantum Electronics, Vol.13, pp. 111-124

Mørk, J.; Tromborg, B. \& Christiansen, P.L. (1988). Bistability and Low-Frequency Fluctuations in Semiconductor Lasers with Optical Feedback: A Theoretical Analysis. IEEE Journal of Quantum Electronics, Vol.24, No.2, pp. 123-133

O’Brien, D.; Hegarty, S.P.; Huyet, G.; McInerney, J.G.; Kettler, T.; Laemmlin, M.; Bimberg, D.; Ustinov, V.M.; Zhukov, A.E.; Mikhrin, S.S. \& Kovsh, A.R. (2003). Feedback Sensitivity of $1.3 \mu \mathrm{m}$ InAs/GaAs Quantum Dot Lasers. Electronics Letters, Vol.39, pp. $1819-1820$

Sano, T. (1994). Antimode Dynamics and Chaotic Itinerancy in the Coherence Collapse of Semiconductor Lasers with Optical Feedback. Physical Review A, Vol.50, No.3, pp. 2719-2726

Schunk, N. \& Petermann, K. (1988). Numerical Analysis of the Feedback Regimes for a Single-Mode Semiconductor Laser with External Feedback. IEEE Journal of Quantum Electronics, Vol.24, No.7, pp. 1242-1247

Tromborg, B.; Osmundsen, J.H. \& Olesen, H. (1984). Stability Analysis for a Semiconductor Laser in an External Cavity. IEEE Journal of Quantum Electronics, Vol.QE-20, No.9, pp. 1023-1032

Tromborg, B. \& Mørk, J. (1990). Nonlinear Injection Locking Dynamics and the Onset of Coherence Collapse in External Cavity Lasers. IEEE Journal of Quantum Electronics, Vol.26, No.4, pp. 642-654

Zamora-Munt, J.; Masoller, C. \& García-Ojalvo, J. (2010). Transient Low-Frequency Fluctuations in Semiconductor Lasers with Optical Feedback. Physical Review A, Vol.81, No.033820

Zou, Q.; Merghem, K.; Azouigui, S.; Martinez, A.; Accard, A.; Chimot, N.; Lelarge, F. \& Ramdane, A. (2010). Feedback-Resistant P-Type Doped InAs/InP Quantum-Dash DFB Lasers for Isolator-Free $10 \mathrm{~Gb} / \mathrm{s}$ Transmission at $1.55 \mu \mathrm{m}$. Appl. Phys. Lett., Vol.97, 231115 


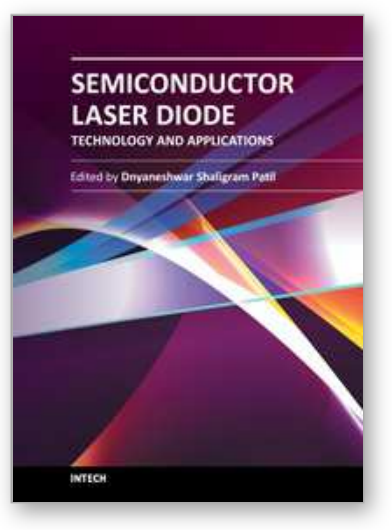

\author{
Semiconductor Laser Diode Technology and Applications \\ Edited by Dr. Dnyaneshwar Shaligram Patil
}

ISBN 978-953-51-0549-7

Hard cover, 376 pages

Publisher InTech

Published online 25, April, 2012

Published in print edition April, 2012

This book represents a unique collection of the latest developments in the rapidly developing world of semiconductor laser diode technology and applications. An international group of distinguished contributors have covered particular aspects and the book includes optimization of semiconductor laser diode parameters for fascinating applications. This collection of chapters will be of considerable interest to engineers, scientists, technologists and physicists working in research and development in the field of semiconductor laser diode, as well as to young researchers who are at the beginning of their career.

\title{
How to reference
}

In order to correctly reference this scholarly work, feel free to copy and paste the following:

Qin Zou and Sheherazade Azouigui (2012). Analysis of Coherence-Collapse Regime of Semiconductor Lasers Under External Optical Feedback by Perturbation Method, Semiconductor Laser Diode Technology and Applications, Dr. Dnyaneshwar Shaligram Patil (Ed.), ISBN: 978-953-51-0549-7, InTech, Available from: http://www.intechopen.com/books/semiconductor-laser-diode-technology-and-applications/analysis-ofcoherence-collapse-regime-of-semiconductor-lasers-under-external-optical-feedback-by-per

\section{INTECH}

open science | open minds

\section{InTech Europe}

University Campus STeP Ri

Slavka Krautzeka 83/A

51000 Rijeka, Croatia

Phone: +385 (51) 770447

Fax: +385 (51) 686166

www.intechopen.com

\section{InTech China}

Unit 405, Office Block, Hotel Equatorial Shanghai

No.65, Yan An Road (West), Shanghai, 200040, China

中国上海市延安西路65号上海国际贵都大饭店办公楼405单元

Phone: +86-21-62489820

Fax: $+86-21-62489821$ 
(C) 2012 The Author(s). Licensee IntechOpen. This is an open access article distributed under the terms of the Creative Commons Attribution 3.0 License, which permits unrestricted use, distribution, and reproduction in any medium, provided the original work is properly cited. 